\title{
Mutations within the HBc Gene of the Hepatitis B Virus: A Study on Iranian Patients
}

\author{
MOHAMMAD ZARE-BIDAKI ${ }^{1}$, FATEMEH AYOOBI ${ }^{2}$, \\ GHOLAMHOSSEIN HASSANSHAHI ${ }^{3}$, MOHAMMAD KAZEMI ARABABADI ${ }^{1}$, \\ TAYEBEH MIRZAEI ${ }^{4}$, AHMAD SHEBANIZADE DAREHDORI ${ }^{5}$, DEREK KENNEDY ${ }^{6}$ \\ ${ }^{1}$ Immunology of Infectious Diseases Research Center, Rafsanjan University of Medical Sciences, Rafsanjan, Iran \\ ${ }^{2}$ Physiology - Pharmacology Research Center, Rafsanjan University of Medical Sciences, Rafsanjan, Iran \\ ${ }_{3}^{3}$ Molecular Medicine Research Center, Rafsanjan University of Medical Sciences, Rafsanjan, Iran. \\ ${ }^{4}$ Department of Nursing, School of Nursing and Midwifery, Rafsanjan University of Medical Sciences, Rafsanjan, Iran \\ ${ }^{5}$ Department of Anatomy, Faculty of Medicine, Rafsanajan University of Medical Sciences, Rafsanjan, Iran \\ ${ }^{6}$ School of Biomolecular and Physical Science, Eskitis Institute for Drug Discovery, Griffith University Nathan, Queensland-Australia
}

\begin{abstract}
SUMMARY
Background: Hepatitis B virus (HBV) is a serious risk factor for several severe liver diseases such as cirrhosis and hepatocellular carcinoma. HBV, like other viruses, uses several mechanisms to escape from specific immune responses including the use of mutations in the genome which lead to epitope variations. There are several immune responses, including $\mathrm{T}$ helper cells, cytotoxic $\mathrm{T}$ lymphocytes, and $\mathrm{B}$ cells, against the core antigen of $\mathrm{HBV}$ (HBcAg) that can lead to HBV eradication. Therefore, mutations within the HBc gene can lead to escape from immune responses by HBV and, hence, understanding the prevalence of $\mathrm{HBc}$ mutations among a specific population can be helpful for future treatment and vaccination. This review addresses the recent information regarding the prevalence of mutations within the $\mathrm{HBc}$ gene among Iranian $\mathrm{HBV}$ infected patients.

Methods: The data presented here was collected gene sequences reported from Iran to the NCBI nucleotide Gen Bank.

Results: Results showed that the prevalence of $\mathrm{HBc}$ gene mutations is frequent in Iranian $\mathrm{HBV}$ infected patients. Conclusions: Based on our searches it seems that escape from immune responses is a plausible reason for the high prevalence of $\mathrm{HBc}$ gene mutations among Iranian HBV infected patients.
\end{abstract}

(Clin. Lab. 2014;60:9-21. DOI: 10.7754/Clin.Lab.2013.130206)

\section{KEY WORDS}

hepatitis $\mathrm{B}$ infection, $\mathrm{HBc}$ gene, mutation

\section{LIST OF ABBREVIATIONS}

HBV - hepatitis B virus

$\mathrm{HBcAg}$ - hepatitis B core antigen

HBsAg - hepatitis B surface antigen

OBI - occult HBV infection

NCBI - National Center for Biotechnology Information

CTL - cytotoxic T lymphocyte

HLA - human leukocyte antigen

MHC - major histocompatibility complex

$\mathrm{CD}$ - cluster of differentiation

\section{INTRODUCTION}

Hepatitis B virus (HBV) is a main cause of human liver diseases [1-3]. Dysfunction and diseases of liver cells including cirrhosis and hepatocellular carcinoma are the most frequent clinical presentations of hepatitis B $[4,5]$. Recent studies revealed that many patients suffer from long term forms of HBV infection including chronic, asymptomatic, and occult (OBI) hepatitis B infection [6-9].

It appears that the quality and quantity of immune responses against HBV infection determines the fate of infection [9]. It has been shown that HBV may alter specific immune responses through a process of selec-

Review Article accepted February 17, 2013 
tive advantage, meaning that an efficient and complete response to wild-type $\mathrm{HBV}$ may result in eradication from the host whereas a cell infected by HBV, harboring a specific mutation, may escape the host's immune system [10], hence the term 'escape mutation'. This could lead to a long term infection of cells by the mutant-containing HBV. The HBc gene encodes the hepatitis core antigen $(\mathrm{HBcAg})$ which is the epitope that elicits several immune cell responses including those by $\mathrm{T}$ helper cells (which respond to $\mathrm{HBcAg}$ amino acids 35 - 45 and 49 - 69), cytotoxic T lymphocytes (HBcAg 18 - 27), and B cells (HBcAg 76 - 87 and 105 - 116) [11, 12]. Escape mutations within $\mathrm{HBcAg}$ epitopes potentially allow HBV to avoid detection and clearance by the host immune system and may represent a mechanism through which infections may persist in some patients. It is worthy to mention that the $\mathrm{D}$ genotype is a unique genotype of HBV present in Iran [13]. Hence, it seems that overall genotype variation is not important in the alteration of immune responses among Iranian patients and that specific mutations within the HBV genome, especially in the $\mathrm{HBc}$ gene, can be the main viral factor for attenuating immune responses and allowing infection to persist. Therefore, this review draws on current reports regarding $\mathrm{HBc}$ gene mutations of $\mathrm{HBV}$ isolated from different clinical presentations of Iranian patients suffering from hepatitis B to find the relationship between reported mutations and the outcome of the infection. In order to more comprehensively report the mutation status in the $\mathrm{HBc}$ gene, the authors have included a search of the reported gene sequences from Iran in the NCBI nucleotide Gen Bank.

\section{Mutations in HBcAg from amino acids 18 to 27 (the cytotoxic $\mathrm{T}$ lymphocyte response region)}

Immune responses by cytotoxic CD8 positive $\mathrm{T}$ cells to viral infections are the main arm of immune responses that can lead to the complete eradication of viral infections [14]. Previous studies revealed that the amino acids from 18 to 27 of $\mathrm{HBcAg}$ are the main region that is recognized by cytotoxic CD8 positive $\mathrm{T}$ cells [15]. Therefore, mutations in this region can lead to attenuated cell cytotoxicity responses and, subsequently, persistent HBV infection can occur [16]. Several researchers from Iran have evaluated the mutations in the epitope from amino acids 18 to 27 of $\mathrm{HBcAg}$ including our previous study on asymptomatic HBV infected patients which reported that there were no mutations found in this epitope of $\mathrm{HBcAg}$ [11]. However, in our search of the GenBank we found a study which reported three mutations T21S, A26S (Gene ID (GI): 54125538), and A21S (GI: 34452647) in this region in chronic hepatitis $\mathrm{B}$ infected patients. In addition, other mutations were reported by other researchers as follow: H21S (GI: 190351525), I27V (GI: 190351480), Q21S (GI: 190351471), G21S (GI: 190351246), N21S (GI: 190351182), Y23F (GI: 190351144), Y24F (GI: 190351144). In addition to the mentioned mutations, Mohebi et al. added another mutation, V21S (GI:
340748298) within the epitope of HBcAg. However, these mutations were also reported by other researchers, but they included the additional mutations M21S (GI: 289598367), S19L (GI: 289598275), and L27V (GI: 289598154). The frequency of mutations targeting Ser21 suggests that this amino acid plays an important role for CTL immune responses. Interestingly, Sendi et al. also demonstrated that Ser21 was the most variable amino acid and that it was substituted by seven different amino acids [16]. It is worthy to mention that the epitope from amino acids 18 to 27 is antigen-presented by HLA-A2 molecules and, hence, this region of $\mathrm{HBcAg}$ would typically be recognized by immune cells of HLA-A2 positive patients. In our studies we evaluated the patients with respect to HLA-A2 but no mutations were found in the HLA-A2 positive patients. Moreover, all of the studies mentioned above, with the exception of Sendi et al., did not evaluate HLA-A2 status in the studied patients. Therefore, we cannot rule out the possibility that the reason for long term infection in those reported studies was not caused by mutations in the antigen presentation genes.

\section{Mutations in HBcAg from amino acids 35 - 45 and 49 - 69 (the $T$ helper lymphocyte response regions)}

$\mathrm{T}$ helper cells are the main cells responsible for cytokine production and co-stimulatory molecule expression which assists humoral and cellular immunity against viral infections $[17,18]$. Previous studies demonstrated that amino acids from 35 to 45 and 49 to 69 with $\mathrm{HBcAg}$ can be presented by type 2 MHC antigen presenting cells to T helper cells $[16,19]$. Activated T helper cells can help B lymphocytes to induce isotype switching and affinity maturation [20,21]. T helpers can also help $\mathrm{T}$ cytotoxic cells to eradicate viral infections such as HBV [22,23]. Therefore, mutations in these regions can lead to defective $\mathrm{T}$ helper responses and subsequently to compromised humoral and cellular immunity against HBV. Iranian researchers have reported several mutations in the amino acids from 35 to 45 and 49 to 69 of HBcAg. Our study on asymptomatic HBV infected patients showed that there were four mutations (D64E, I66M, N67T, and S69A) in amino acids from 49 to 69 , while no mutations were found in amino acids from 35 to 45 [11]. According to our search of Gen Bank, Amini-Bavil-Olyaee et al. reported four mutations F38Y(GI: 54125538), A35S (GI: 34452656), S45P (GI: 34452652), and Q40E (GI: 34452640) in the 35 - 45 region and seven mutations S67T (GI: 54125538), D64E (GI: 34452658), F59I (GI: 34452654), I66M plus S69A (GI: 34452652), V55L (GI: 34452638), and T56R (GI: 34452626) in the 49 69 region of chronic hepatitis $\mathrm{B}$ infected patients. Mohebi et al. also have submitted T35S plus D40E (GI: 190351568), N45P (GI: 190351293), and T45P (GI: 190351276) mutations in the amino acids from 35 to 45 and several mutations in amino acids from 49 to 69 as follow: T49S (GI: 190351568), N67T (GI: 190351539), V59I (GI: 190351497), A50P (GI: 190351483), A50H 
(GI:190351414), I55L (GI:190351403), H50P (GI: 190351377), H57Q, V58A plus S59I (GI: 190351322), Q67T (GI: 190351296), C59I (GI: 190351210), and W61C (GI: 190351175). In 2012, these researchers also submitted other $\mathrm{HBc}$ gene sequences with the following mutations: E38Y (GI: 340748227) and G40E (GI: $340748170)$ within the amino acids from 35 to 45 and E58A (GI: 340748457), I60L (GI: 340748298), V56A (GI: 340748263), and S50P (GI: 340748220) within the amino acids from 49 to 69 of $\mathrm{HBcAg}$ [12]. Garmiri et al. reported the existence of the same mutations but added several new ones including P35S (GI: 289598430), Q45P (GI: 289598388), H45P (GI: 289598381), P41I (GI: 289598268), and S41A (GI: 289598189) in amino acids from 35 to 45 and Y59I plus L66M plus G67T (GI: 289598409), A67T (GI: 289598402), T66M (GI: 289598261), F59L (GI: 289598212), and S60L (GI: 289598168) in amino acids from 49 to 69 [24]. F38Y and D40E are the most common mutations within the 35 - 45 region whereas D64E, S69A, T/I66M, and S/N67T are the most common in the 49 - 69 region (see Table 1). Based on these results, it can be concluded that these mutations may play key roles in the escape of $\mathrm{T}$ helper immune responses by $\mathrm{HBV}$. Previous studies revealed that $\mathrm{T}$ helpers induce humoral and cellular immunity against viral infections via expression of co-stimulatory molecules and cytokines [18]. Barboza et al. reported that $\mathrm{T}$ helper cells of chronic HBV infected patients were unable to increase the expression of CD40L as the main co-stimulatory molecule for macrophage and B lymphocyte activation [25]. Several studies also demonstrated that cytokine production by $\mathrm{T}$ helper cells was decreased in hepatitis $\mathrm{B}$ infected patients $[8,9,26]$. Therefore, it may be concluded that HBV can escape recognition by $\mathrm{T}$ helper lymphocytes especially when they harbor the F38Y and D40E mutations in the $35-45$ region and D64E, S69A, $\mathrm{T} / \mathrm{I} 66 \mathrm{M}$, and $\mathrm{S} / \mathrm{N} 67 \mathrm{~T}$ in the 49 - 69 region.

\section{Mutations in HBcAg from amino acids $76-87$ and 105 - 116 (the $B$ cell lymphocyte response regions)}

Humoral immunity is another arm of immune responses, especially in the protection against viral infection $[27,28]$. Anti-HBs are protective antibodies that are produced against HBsAg (HBV vaccination components) [29]. Anti-HBc is produced against amino acids $76-87$ as well as to the $105-116$ region of $\mathrm{HBcAg}$ [30]. Furthermore, humoral immunity is considered one of the main immune responses against HBV and therefore mutations within the $\mathrm{B}$ cell lymphocyte epitope recognition region of $\mathrm{HBcAg}$ can be utilized by $\mathrm{HBV}$ to escape from these immune responses [31,32]. Interestingly, we have shown previously that there were three mutations in the 76 - 87 region (Q77E, T80I, N87S) and one mutation in the 105 - 116 region (L116I) of asymptomatic HBV infected patients [11]. Like other HBc gene regions, our search of GenBank revealed that Amini-Bavil-Olyaee et al. found five mutations Q79P, Q80I
(GI: 54125538), T80I (GI: 34452658), V80I (GI: 34452656), and M85V (GI: 34452631) in the76 - 87 region as well as E113D (GI: 54125538), L116I (GI: 34452654), T105I (GI: 34452652), and N113E (GI: $34452624)$ in the 105 - 116 region of chronic hepatitis B infected patients.

Additionally, other mutations were reported by Mohebi et al. which included G87S (GI: 190351556), Q77E, V114T (GI: 190351536), Q84E (GI: 190351525), A80I (GI: 190351501), E83D (GI: 190351486), I86V (GI: 190351480), P80I (GI: 190351417), Q87S (GI: 190351210), T87S (GI: 190351199), and I85V plus N87S (GI: 190351156) in the $76-87$ region and P113E (GI: 190351556), I114T (GI: 190351539), V105I plus M109T (GI: 190351525), Q113E (GI: 190351522), A109T (GI: 190351497), V114T (GI: 190351486), V116I (GI: 190351471), S113E (GI: 190351417), L105I (GI: 190351414), T116I (GI: 190351377), S116I (GI: 190351347), S109T (GI: 190351325), K111R (GI: 190351259), and K112R (GI: 190351175) in the amino acids from 105 to 116 . Mohebi et al. also reported that the mentioned mutations were also found in their HBV chronic patients. In addition, they also reported mutations including N78D (GI: 340748661), N67T (GI: 340748592), D77E (GI: 340748256), and A85V (GI: 340748185) within the $76-87$ region and I109T (GI: 340748675), Y107C (GI: 340748585), and M119T (GI: 340748213 ) within the amino acids from 105 to 116 of $\mathrm{HBcAg}$. Although, these mutations were also reported by Garmiri et al. they also included mutations such as R87S (GI: 289598402) and E84D (GI: 289598317) in the 76 - 87 region and M114T (GI: 289598409) and N109T (GI: 289598212) in the 105 - 116 region in their report. The most common mutations in the epitope recognized by B cell lymphocytes, in the region from 76 to 87 were those changing Ile80 (Table 1). Ise80 was replaced by five different amino acids including Glutamine, Proline, Alanine, Threonine and Valine. D/Q77E, $\mathrm{A} / \mathrm{I} / \mathrm{M} 85 \mathrm{~V}, \mathrm{M} / \mathrm{I} / \mathrm{V} 114 \mathrm{~T}$ and $\mathrm{R} / \mathrm{T} / \mathrm{Q} / \mathrm{G} / \mathrm{N} 87 \mathrm{~S}$ mutations were also reported but with less frequency than mutations in position Ile80. Therefore, it appears that mutations at Glu77, Val85, Ser87, Thr114 and especially Ile 80 were seen repeatedly in the B cell lymphocyte epitope recognition region of the $\mathrm{HBc}$ gene in HBV infected Iranian patients and these mutations may be a mechanism employed by the virus to escape antibody recognition.

\section{CONCLUSION}

According to the reports mentioned above, it seems that the prevalence of $\mathrm{HBc}$ gene mutations is frequent in Iranian HBV infected patients. Escape from immune responses is a plausible reason for the high prevalence of $\mathrm{HBc}$ gene mutations among Iranian HBV infected patients. Moreover, the studies mentioned above are in reference to the mutations within the $\mathrm{HBc}$ gene of 
Table 1. The table reports the amino acid substitutions found in the five epitopes (CTL recognition epitope from amino acids 18 - 27, T helper recognition epitopes from 35 - 45 and 49 - 69 and B cell recognition epitopes 76 - 87 and 105 - 116) of the hepatitis $B$ core antigen. Substitutions are classified by the epitope they are found in. Also shown are the GenBank gene identifiers and the references in which they are reported. Amino Acids are represented using the single letter code and numbers represent the position of the amino acid within the $\mathrm{HBc}$ antigen protein.

\begin{tabular}{|c|c|c|c|c|c|c|}
\hline \multicolumn{5}{|c|}{ Status of mutation in antigenic epitopes of the $\mathrm{HBV}$ core protein } & \multirow{2}{*}{$\begin{array}{c}\text { NCBI } \\
\text { GenBank } \\
\text { Gene ID }\end{array}$} & \multirow[b]{2}{*}{ Reference } \\
\hline $\begin{array}{c}\text { CTL } \\
\text { (HBcAg } 18 \text { - 27) }\end{array}$ & $\begin{array}{c}\text { T helper } \\
\text { (HBcAg } 35-45)\end{array}$ & $\begin{array}{c}\text { T helper } \\
\text { (HBcAg } 49-69)\end{array}$ & $\begin{array}{c}\text { B cell } \\
(\text { HBcAg } 76-87)\end{array}$ & $\begin{array}{c}\text { B cell } \\
(\text { HBcAg } 105-116)\end{array}$ & & \\
\hline NF & NF & $\begin{array}{l}\text { D64E } \\
\text { I66M } \\
\text { N67T } \\
\text { S69A }\end{array}$ & $\begin{array}{c}\text { Q77E } \\
\text { T80I } \\
\text { N87S }\end{array}$ & L116I & $\begin{array}{c}\text { No } \\
\text { submitted }\end{array}$ & [11] \\
\hline \multirow[t]{5}{*}{$\begin{array}{r}\text { T21S } \\
\text { A26S } \\
\end{array}$} & F38Y & S67T & $\begin{array}{l}\text { Q79P } \\
\text { Q80I }\end{array}$ & E113D & 54125538 & [34] \\
\hline & & D64E & T80I & & 34452658 & [35] \\
\hline & A35S & D64E & V80I & & 34452656 & [35] \\
\hline & & F59I & T80I & L116I & 34452654 & [35] \\
\hline & S45P & $\begin{array}{l}\text { D64E } \\
\text { I66M } \\
\text { S69A }\end{array}$ & T80I & $\begin{array}{l}\text { T105I } \\
\text { D113E }\end{array}$ & 34452652 & [35] \\
\hline A21S & & & T80I & $\begin{array}{c}\text { L116I } \\
\text { D113E }\end{array}$ & 34452647 & [35] \\
\hline A21S & $\begin{array}{l}\text { F38Y } \\
\text { Q40E }\end{array}$ & $\begin{array}{l}\text { D64E } \\
\text { S67T }\end{array}$ & T80I & & 34452640 & [35] \\
\hline \multirow[t]{7}{*}{ T21S } & & V55L & T80I & L116I & 34452638 & [35] \\
\hline & & $\begin{array}{l}\text { D64E } \\
\text { I66M } \\
\text { S69A }\end{array}$ & T80I & & 34452636 & [35] \\
\hline & & I66M & $\begin{array}{c}\text { T80I } \\
\text { M85V }\end{array}$ & $\begin{array}{l}\text { L116I } \\
\text { D113E }\end{array}$ & 34452631 & [35] \\
\hline & & T56R & & & 34452626 & [35] \\
\hline & & $\begin{array}{l}\text { D64E } \\
\text { I66M }\end{array}$ & V80I & N113E & 34452624 & [35] \\
\hline & $\begin{array}{l}\text { T35S } \\
\text { D40E }\end{array}$ & T49S & & & 190351568 & [36] \\
\hline & D40E & & T80I & & 190351563 & [36] \\
\hline A21S & $\begin{array}{l}\text { F38Y } \\
\text { D40E } \\
\end{array}$ & $\begin{array}{l}\text { D64E } \\
\text { S67T } \\
\end{array}$ & $\begin{array}{c}\text { T80I } \\
\text { G87S } \\
\end{array}$ & P113E & 190351556 & [36] \\
\hline A21S & $\begin{array}{l}\text { F38Y } \\
\text { D40E }\end{array}$ & & & L116I & 190351550 & [36] \\
\hline \multirow[t]{2}{*}{ T21S } & & $\begin{array}{l}\text { D64E } \\
\text { N67T }\end{array}$ & T80I & I114T & 190351539 & {$[36]$} \\
\hline & & $\begin{array}{l}\text { D64E } \\
\text { I66M } \\
\text { N67T } \\
\text { S69A } \\
\end{array}$ & $\begin{array}{c}\text { Q77E } \\
\text { T80I } \\
\text { V114T }\end{array}$ & & 190351536 & [36] \\
\hline \multirow[t]{6}{*}{ H21S } & D40E & $\begin{array}{l}\text { D64E } \\
\text { N67T }\end{array}$ & $\begin{array}{c}\text { Q77E } \\
\text { T80I } \\
\text { Q84E }\end{array}$ & $\begin{array}{l}\text { V105I } \\
\text { M109T }\end{array}$ & 190351525 & [36] \\
\hline & F38Y & & $\begin{array}{c}\text { D77E } \\
\text { T80I }\end{array}$ & Q113E & 190351522 & [36] \\
\hline & & D64E & T80I & & 190351515 & [36] \\
\hline & & D64E & V80I & & 190351508 & [36] \\
\hline & & D64E & V80I & & 190351505 & [36] \\
\hline & & D64E & A80I & & 190351501 & [36] \\
\hline
\end{tabular}




\begin{tabular}{|c|c|c|c|c|c|c|}
\hline & & V59I & T80I & $\begin{array}{l}\text { A109T } \\
\text { Q113E }\end{array}$ & 190351497 & [36] \\
\hline & & $\begin{array}{l}\text { D64E } \\
\text { I66M } \\
\text { S69A }\end{array}$ & T80I & & 190351493 & {$[36]$} \\
\hline & & D64E & V80I & & 190351490 & {$[36]$} \\
\hline $\begin{array}{l}\text { T21S } \\
\text { A26S } \\
\end{array}$ & & $\begin{array}{l}\text { D64E } \\
\text { N67T } \\
\end{array}$ & $\begin{array}{r}\text { V80I } \\
\text { E83D } \\
\end{array}$ & V114T & 190351486 & {$[36]$} \\
\hline A21S & F38Y & $\begin{array}{l}\text { A50P } \\
\text { D64E } \\
\text { I66M } \\
\text { S69A } \\
\end{array}$ & T80I & L116I & 190351483 & {$[36]$} \\
\hline $\mathrm{I} 27 \mathrm{~V}$ & $\begin{array}{l}\text { A35S } \\
\text { D40E }\end{array}$ & $\begin{array}{l}\text { A50P } \\
\text { D64E } \\
\text { S67T }\end{array}$ & $\begin{array}{c}\text { V80I } \\
\text { E83D } \\
\text { I86V } \\
\text { G87S } \\
\end{array}$ & $\begin{array}{c}\text { M109T } \\
\text { V116I }\end{array}$ & 190351480 & [36] \\
\hline \multirow[t]{2}{*}{ T21S } & & T49S & $\begin{array}{c}\text { D77E } \\
\text { T80I } \\
\end{array}$ & D113E & 190351477 & [36] \\
\hline & D40E & V59I & T80I & & 190351474 & [36] \\
\hline \multirow[t]{13}{*}{ Q21S } & & & T80I & V116I & 190351471 & [36] \\
\hline & D40E & D64E & $\begin{array}{l}\text { D77E } \\
\text { V80I }\end{array}$ & & 190351468 & {$[36]$} \\
\hline & F38Y & & & & 190351462 & {$[36$} \\
\hline & D40E & & T80I & L116I & 190351459 & [36] \\
\hline & F38Y & & & & 190351456 & {$[36]$} \\
\hline & & D64E & $\begin{array}{l}\text { Q77E } \\
\text { V80I }\end{array}$ & D113E & 190351453 & {$[36]$} \\
\hline & & & T80I & D113E & 190351450 & [36] \\
\hline & & D64E & A80I & & 190351447 & [36] \\
\hline & D40E & $\begin{array}{l}\text { T49S } \\
\text { D64E } \\
\text { N67T } \\
\end{array}$ & $\begin{array}{r}\text { V80I } \\
\text { G87S }\end{array}$ & $\begin{array}{l}\text { Q113E } \\
\text { V116I }\end{array}$ & 190351443 & {$[36]$} \\
\hline & D40E & $\begin{array}{l}\text { D64E } \\
\text { I66M } \\
\text { S69A } \\
\end{array}$ & T80I & & 190351436 & {$[36]$} \\
\hline & & T49S & V80I & L116I & 190351433 & [36] \\
\hline & & $\begin{array}{l}\text { D64E } \\
\text { I66M } \\
\text { S69A }\end{array}$ & T80I & Q113E & 190351427 & [36] \\
\hline & & $\begin{array}{l}\text { V59I } \\
\text { D64E }\end{array}$ & V80I & V116I & 190351420 & [36] \\
\hline \multirow[t]{2}{*}{ H21S } & & N67T & P80I & $\begin{array}{l}\text { S113E } \\
\text { L116I } \\
\end{array}$ & 190351417 & {$[36]$} \\
\hline & $\begin{array}{l}\text { F38Y } \\
\text { D40E } \\
\end{array}$ & A50H & & $\begin{array}{l}\text { L105I } \\
\text { D113E }\end{array}$ & 190351414 & [36] \\
\hline \multirow[t]{2}{*}{ T21S } & & $\begin{array}{l}\text { I55L } \\
\text { S67T }\end{array}$ & T80I & $\begin{array}{l}\text { M109T } \\
\text { D113E } \\
\text { L116I }\end{array}$ & 190351403 & {$[36]$} \\
\hline & & & T80I & $\begin{array}{l}\text { V105I } \\
\text { V114T } \\
\end{array}$ & 190351400 & [36] \\
\hline \multirow[t]{2}{*}{ A21S } & & $\begin{array}{l}\text { D64E } \\
\text { I66M } \\
\text { S69A } \\
\end{array}$ & T80I & & 190351397 & {$[36]$} \\
\hline & D40E & T49S & $\begin{array}{c}\text { Q77E } \\
\text { T80I }\end{array}$ & & 190351394 & {$[36]$} \\
\hline
\end{tabular}


M. Z. BIDAKI et al.

\begin{tabular}{|c|c|c|c|c|c|c|}
\hline & & $\begin{array}{l}\text { T49S } \\
\text { D64E } \\
\text { I66M } \\
\text { S69A } \\
\end{array}$ & T80I & L116I & 190351391 & [36] \\
\hline & & & T80I & V114T & 190351380 & [36] \\
\hline & & H50P & T80I & $\begin{array}{c}\text { D113E } \\
\text { T116I }\end{array}$ & 190351377 & [36] \\
\hline & & D64E & T80I & & 190351374 & [36] \\
\hline & & & & D113E & 190351363 & [36] \\
\hline & & $\begin{array}{l}\text { D64E } \\
\text { I66M } \\
\text { S69A }\end{array}$ & T80I & L116I & 190351360 & [36] \\
\hline & $\begin{array}{l}\text { F38Y } \\
\text { D40E }\end{array}$ & H50P & $\begin{array}{c}\text { D77E } \\
\text { T80I }\end{array}$ & L116I & 190351357 & [36] \\
\hline \multirow[t]{2}{*}{ Q21S } & $\begin{array}{l}\text { F38Y } \\
\text { D40E } \\
\end{array}$ & & T80I & $\begin{array}{l}\text { L105I } \\
\text { D113E } \\
\end{array}$ & 190351354 & [36] \\
\hline & & & & S116I & 190351347 & [36] \\
\hline \multirow[t]{5}{*}{ A21S } & & $\begin{array}{l}\text { D64E } \\
\text { I66M } \\
\text { S69A }\end{array}$ & T80I & Q113E & 190351344 & [36] \\
\hline & & $\begin{array}{l}\text { D64E } \\
\text { I66M } \\
\text { S69A }\end{array}$ & T80I & $\begin{array}{l}\text { D113E } \\
\text { V116I }\end{array}$ & 190351341 & [36] \\
\hline & & N67T & T80I & & 190351328 & [36] \\
\hline & D40E & & T80I & S109T & 190351325 & [36] \\
\hline & & $\begin{array}{c}\text { H57Q } \\
\text { V58A } \\
\text { S59I } \\
\text { D64E } \\
\text { N67T }\end{array}$ & & L116I & 190351322 & [36] \\
\hline A21S & T35S & $\begin{array}{l}\text { D64E } \\
\text { S67T } \\
\end{array}$ & T80I & $\begin{array}{c}\text { M109T } \\
\text { V116I } \\
\end{array}$ & 190351319 & [36] \\
\hline \multirow[t]{2}{*}{ T21S } & & T49S & $\begin{array}{c}\text { D77E } \\
\text { T80I } \\
\end{array}$ & $\begin{array}{l}\text { V105I } \\
\text { V116I } \\
\end{array}$ & 190351312 & [36] \\
\hline & & D64E & V80I & & 190351304 & [36] \\
\hline \multirow[t]{5}{*}{ A21S } & & $\begin{array}{l}\text { A50P } \\
\text { Q67T }\end{array}$ & $\begin{array}{c}\text { D77E } \\
\text { T80I } \\
\text { G87S } \\
\end{array}$ & $\begin{array}{l}\text { V105I } \\
\text { V114T } \\
\text { L116I } \\
\end{array}$ & 190351296 & [36] \\
\hline & $\begin{array}{l}\text { F38Y } \\
\text { D40E } \\
\text { N45P }\end{array}$ & D64E & T80I & & 190351293 & [36] \\
\hline & & F59I & T80I & L116I & 190351282 & [36] \\
\hline & F38Y & & T80I & T116I & 190351279 & [36] \\
\hline & $\begin{array}{l}\text { D40E } \\
\text { T45P }\end{array}$ & $\begin{array}{l}\text { D64E } \\
\text { I66M } \\
\text { S69A }\end{array}$ & $\begin{array}{c}\text { D77E } \\
\text { T80I }\end{array}$ & D113E & 190351276 & [36] \\
\hline \multirow[t]{4}{*}{ A21S } & $\begin{array}{l}\text { T35S } \\
\text { D40E } \\
\end{array}$ & D64E & V80I & V116I & 190351273 & [36] \\
\hline & & D64E & $\begin{array}{c}\text { T80I } \\
\text { E83D } \\
\text { G87S }\end{array}$ & & 190351270 & [36] \\
\hline & $\begin{array}{l}\text { F38Y } \\
\text { N45P } \\
\end{array}$ & & T80I & K111R & 190351259 & [36] \\
\hline & T35S & $\begin{array}{l}\text { D64E } \\
\text { I66M } \\
\text { S69A }\end{array}$ & T80I & L116I & 190351256 & [36] \\
\hline T21S & A35S & $\begin{array}{l}\text { T49S } \\
\text { I60L }\end{array}$ & $\begin{array}{c}\text { T80I } \\
\text { G87S }\end{array}$ & & 190351253 & [36] \\
\hline
\end{tabular}




\begin{tabular}{|c|c|c|c|c|c|c|}
\hline G21S & & $\begin{array}{l}\text { D64E } \\
\text { S67T } \\
\end{array}$ & T80I & Q113E & 190351246 & {$[36]$} \\
\hline T21S & F38Y & T49S & T80I & & 190351243 & {$[36]$} \\
\hline \multirow[t]{6}{*}{ T21S } & & D64E & V80I & & 190351240 & [36] \\
\hline & & $\begin{array}{l}\text { D64E } \\
\text { N67T } \\
\end{array}$ & V80I & & 190351237 & {$[36$} \\
\hline & & $\begin{array}{l}\text { D64E } \\
\text { S67T } \\
\end{array}$ & T80I & & 190351230 & {$[36]$} \\
\hline & & $\begin{array}{l}\text { D64E } \\
\text { I66M } \\
\text { S69A }\end{array}$ & T80I & & 190351227 & {$[36]$} \\
\hline & $\begin{array}{l}\text { A35S } \\
\text { F38Y } \\
\text { D40E }\end{array}$ & $\begin{array}{l}\text { T49S } \\
\text { S67T }\end{array}$ & T80I & & 190351223 & {$[36]$} \\
\hline & & T49S & & & 190351216 & {$[36]$} \\
\hline \multirow[t]{3}{*}{ A21S } & & & T80I & $\begin{array}{l}\text { D113E } \\
\text { V116I }\end{array}$ & 190351213 & {$[36]$} \\
\hline & & C59I & $\begin{array}{l}\text { Q79P } \\
\text { A80I } \\
\text { Q87S }\end{array}$ & $\begin{array}{l}\text { D113E } \\
\text { I114T }\end{array}$ & 190351210 & {$[36]$} \\
\hline & & V59I & $\begin{array}{l}\text { Q77E } \\
\text { V80I } \\
\end{array}$ & I114T & 190351207 & {$[36]$} \\
\hline \multirow[t]{4}{*}{ A21S } & F38Y & $\begin{array}{r}\text { T49S } \\
\text { N67T }\end{array}$ & $\begin{array}{c}\text { Q79P } \\
\text { T80I } \\
\text { T87S } \\
\end{array}$ & V105I & 190351199 & {$[36]$} \\
\hline & & D64E & V80I & & 190351196 & {$[36]$} \\
\hline & & & V80I & L116I & 190351193 & [36] \\
\hline & & & T80I & Q113E & 190351189 & [36] \\
\hline A21S & F38Y & $\begin{array}{l}\text { V59I } \\
\text { D64E } \\
\end{array}$ & V80I & V116I & 190351185 & {$[36]$} \\
\hline \multirow[t]{3}{*}{ N21S } & $\begin{array}{l}\text { F38Y } \\
\text { D40E }\end{array}$ & $\begin{array}{l}\text { I55L } \\
\text { D64E }\end{array}$ & $\begin{array}{l}\text { Q79P } \\
\text { A80I }\end{array}$ & V105I & 190351182 & {$[36]$} \\
\hline & & W61C & & K112R & 190351175 & [36] \\
\hline & F38Y & $\begin{array}{l}\text { D64E } \\
\text { I66M } \\
\text { S69A } \\
\end{array}$ & & D113E & 190351171 & {$[36]$} \\
\hline A21S & F38Y & $\begin{array}{l}\text { D64E } \\
\text { I66M } \\
\text { S69A }\end{array}$ & T80I & $\begin{array}{l}\text { V114T } \\
\text { L116I }\end{array}$ & 190351168 & {$[36]$} \\
\hline \multirow[t]{5}{*}{ T21S } & D40E & T49S & $\begin{array}{l}\text { Q77E } \\
\text { V80I } \\
\end{array}$ & V114T & 190351165 & {$[36]$} \\
\hline & & D64E & T80I & & 190351162 & {$[36$} \\
\hline & & D64E & T80I & & 190351159 & [36] \\
\hline & D40E & $\begin{array}{l}\text { D64E } \\
\text { I66M } \\
\text { N67T } \\
\text { S69A } \\
\end{array}$ & $\begin{array}{l}\text { Q79P } \\
\text { T80I } \\
\text { I85V } \\
\text { N87S } \\
\end{array}$ & & 190351156 & {$[36]$} \\
\hline & & F59I & T80I & & 190351149 & [36] \\
\hline \multirow[t]{2}{*}{$\begin{array}{l}\text { T21S } \\
\text { Y23F } \\
\text { Y24F } \\
\end{array}$} & & & T80I & Q113E & 190351144 & {$[36]$} \\
\hline & F38Y & D64E & & $\begin{array}{l}\text { D113E } \\
\text { V116I }\end{array}$ & 190351141 & {$[36]$} \\
\hline \multirow[t]{2}{*}{ A21S } & & & $\begin{array}{c}\text { D77E } \\
\text { T80I } \\
\end{array}$ & $\begin{array}{l}\text { Q113E } \\
\text { V116I } \\
\end{array}$ & 190351135 & {$[36]$} \\
\hline & & & $\begin{array}{c}\text { Q77E } \\
\text { T80I }\end{array}$ & $\begin{array}{l}\text { D113E } \\
\text { V116I }\end{array}$ & 190351132 & {$[36]$} \\
\hline
\end{tabular}


M. Z. BIDAKI et al.

\begin{tabular}{|c|c|c|c|c|c|c|}
\hline T21S & & $\begin{array}{l}\text { D64E } \\
\text { N67T } \\
\end{array}$ & $\begin{array}{r}\text { V80I } \\
\text { G87S } \\
\end{array}$ & & 190351129 & [36] \\
\hline & & & N78D & & 190351126 & [36] \\
\hline & & D64E & T80I & & 190351122 & [36] \\
\hline & $\begin{array}{l}\text { F38Y } \\
\text { D40E }\end{array}$ & $\begin{array}{l}\text { T49S } \\
\text { D64E }\end{array}$ & T80I & $\begin{array}{l}\text { I109T } \\
\text { V116I }\end{array}$ & 340748675 & [12] \\
\hline & & $\begin{array}{l}\text { D64E } \\
\text { N67T }\end{array}$ & T80I & I114T & 340748668 & [12] \\
\hline & & & N78D & & 340748661 & [12] \\
\hline & & & T80I & L116I & 340748631 & [12] \\
\hline & & & D77E & Q113E & 340748616 & [12] \\
\hline & & V59I & $\begin{array}{l}\text { Q77E } \\
\text { V80I }\end{array}$ & I114T & 340748608 & [12] \\
\hline & & & $\begin{array}{c}\text { T80I } \\
\text { T87S }\end{array}$ & & 340748600 & [12] \\
\hline & & T49S & $\begin{array}{c}\text { N67T } \\
\text { T80I }\end{array}$ & Q113E & 340748592 & [12] \\
\hline \multirow[t]{24}{*}{ A21S } & F38Y & T49S & $\begin{array}{c}\text { D77E } \\
\text { T80I } \\
\end{array}$ & $\begin{array}{l}\text { Y107C } \\
\text { L116I }\end{array}$ & 340748585 & [12] \\
\hline & & D64E & V80I & & 340748578 & [12] \\
\hline & T35S & D64E & V80I & & 340748554 & [12] \\
\hline & & D64E & & & 340748546 & [12] \\
\hline & & D64E & $\begin{array}{l}\text { T80I } \\
\text { I85V }\end{array}$ & & 340748538 & [12] \\
\hline & & D64E & $\begin{array}{c}\text { T80I } \\
\text { G87S }\end{array}$ & V116I & 340748531 & [12] \\
\hline & D40E & $\begin{array}{l}\text { D64E } \\
\text { I66M } \\
\text { S69A }\end{array}$ & T80I & & 340748524 & [12] \\
\hline & $\begin{array}{l}\text { A35S } \\
\text { F38Y } \\
\end{array}$ & & T80I & & 340748517 & [12] \\
\hline & & T49S & T80I & & 340748478 & [12] \\
\hline & $\begin{array}{l}\text { D40E } \\
\text { S45P } \\
\end{array}$ & D64E & V80I & $\begin{array}{l}\text { M109T } \\
\text { D113E }\end{array}$ & 340748471 & [12] \\
\hline & & $\begin{array}{l}\text { D64E } \\
\text { I66M } \\
\text { S69A } \\
\end{array}$ & A80I & Q113E & 340748464 & [12] \\
\hline & & $\begin{array}{l}\text { E58A } \\
\text { N67T } \\
\end{array}$ & $\begin{array}{r}\text { T80I } \\
\text { N87S } \\
\end{array}$ & & 340748457 & [12] \\
\hline & & $\begin{array}{l}\text { E58A } \\
\text { N67T }\end{array}$ & $\begin{array}{c}\text { Q79P } \\
\text { T80I } \\
\text { N87S }\end{array}$ & & 340748450 & [12] \\
\hline & F38Y & & & & 340748443 & [12] \\
\hline & F38Y & A50P & T80I & & 340748436 & [12] \\
\hline & F38Y & & T80I & $\begin{array}{l}\text { T105I } \\
\text { D113E }\end{array}$ & 340748429 & [12] \\
\hline & & T49S & T80I & & 340748421 & [12] \\
\hline & $\begin{array}{l}\text { T35S } \\
\text { F38Y }\end{array}$ & T49S & T80I & I114T & 340748413 & [12] \\
\hline & & & & V105I & 340748405 & [12] \\
\hline & & & & L105I & 340748381 & [12] \\
\hline & & & T80I & & 340748357 & [12] \\
\hline & & D64E & & & 340748334 & [12] \\
\hline & & D64E & V80I & & 340748319 & [12] \\
\hline & & & V80I & V116I & 340748312 & [12] \\
\hline
\end{tabular}




\begin{tabular}{|c|c|c|c|c|c|c|}
\hline & & $\begin{array}{l}\text { C59I } \\
\text { N67T }\end{array}$ & T80I & Q113E & 340748305 & [12] \\
\hline $\begin{array}{l}\text { V21S } \\
\text { I27V }\end{array}$ & & $\begin{array}{l}\text { I55L } \\
\text { I60L } \\
\text { N67T }\end{array}$ & & V105I & 340748298 & [12] \\
\hline A21S & F38Y & $\begin{array}{r}\text { T49S } \\
\text { N67T }\end{array}$ & $\begin{array}{c}\text { Q79P } \\
\text { T80I } \\
\text { T87S }\end{array}$ & V105I & 340748291 & [12] \\
\hline \multirow[t]{4}{*}{ T21S } & D40E & & $\begin{array}{c}\text { Q79P } \\
\text { T80I }\end{array}$ & & 340748284 & [12] \\
\hline & & & $\begin{array}{c}\text { T80I } \\
\text { T87S }\end{array}$ & L116I & 340748277 & [12] \\
\hline & & D64E & $\begin{array}{l}\text { Q77E } \\
\text { V80I }\end{array}$ & D113E & 340748270 & [12] \\
\hline & & $\begin{array}{c}\text { H57Q } \\
\text { V56A } \\
\text { S59I } \\
\text { D64E } \\
\text { N67T }\end{array}$ & T80I & $\begin{array}{l}\text { D113E } \\
\text { L116I }\end{array}$ & 340748263 & [12] \\
\hline \multirow[t]{4}{*}{ T21S } & & T49S & $\begin{array}{c}\text { D77E } \\
\text { T80I }\end{array}$ & $\begin{array}{l}\text { V105I } \\
\text { D113E }\end{array}$ & 340748256 & [12] \\
\hline & $\begin{array}{l}\text { D40E } \\
\text { T45P }\end{array}$ & $\begin{array}{l}\text { D64E } \\
\text { I66M } \\
\text { S69A }\end{array}$ & $\begin{array}{c}\text { D77E } \\
\text { T80I }\end{array}$ & D113E & 340748249 & [12] \\
\hline & & $\begin{array}{l}\text { D64E } \\
\text { N67T }\end{array}$ & A80I & $\begin{array}{l}\text { D113E } \\
\text { V105I }\end{array}$ & 340748242 & [12] \\
\hline & & $\begin{array}{l}\text { I55L } \\
\text { V59I } \\
\text { S67T }\end{array}$ & T80I & $\begin{array}{l}\text { Q113E } \\
\text { L116I }\end{array}$ & 340748235 & [12] \\
\hline \multirow[t]{4}{*}{ A21S } & E38Y & A50P & T80I & L116I & 340748227 & [12] \\
\hline & & S50P & $\begin{array}{l}\text { V80I } \\
\text { T87S }\end{array}$ & L116I & 340748220 & [12] \\
\hline & T35S & $\begin{array}{l}\text { D64E } \\
\text { I66M } \\
\text { S69A }\end{array}$ & T80I & M119T & 340748213 & [12] \\
\hline & & & T80I & & 340748206 & {$[12]$} \\
\hline \multirow[t]{3}{*}{ G21S } & & T49S & $\begin{array}{c}\text { T80I } \\
\text { T87S }\end{array}$ & & 340748199 & [12] \\
\hline & & T49S & T80I & Q113E & 340748192 & [12] \\
\hline & & N67T & $\begin{array}{c}\text { Q77E } \\
\text { T80I } \\
\text { A85V }\end{array}$ & D113E & 340748185 & [12] \\
\hline T21S & & N67T & $\begin{array}{c}\text { Q77E } \\
\text { T80I } \\
\text { A85V }\end{array}$ & $\begin{array}{l}\text { D113E } \\
\text { V116I }\end{array}$ & 340748178 & [12] \\
\hline A21S & $\begin{array}{c}\text { G40E } \\
\text { S45P }\end{array}$ & $\begin{array}{l}\text { V59I } \\
\text { D64E } \\
\text { I66M } \\
\text { N67T } \\
\text { S69A }\end{array}$ & T80I & V116I & 340748170 & [12] \\
\hline \multirow[t]{6}{*}{ T21S } & & T49S & V80I & & 340748163 & [12] \\
\hline & F38Y & & $\begin{array}{c}\text { D77E } \\
\text { T80I }\end{array}$ & & 340748133 & [12] \\
\hline & & & T80I & & 340748126 & [12] \\
\hline & T35S & D64E & T80I & $\begin{array}{l}\text { V105I } \\
\text { L116I }\end{array}$ & 340748118 & [12] \\
\hline & & $\begin{array}{l}\text { D64E } \\
\text { S67T }\end{array}$ & & & 340748111 & [12] \\
\hline & D40E & D64E & V80I & & 340748105 & {$[12]$} \\
\hline
\end{tabular}


M. Z. BIDAKI et al.

\begin{tabular}{|c|c|c|c|c|c|c|}
\hline & & & T80I & & 340748098 & [12] \\
\hline & & & T80I & L116I & 340748091 & [12] \\
\hline & & T49S & V80I & L116I & $\mathbf{3 4 0 7 4 8 0 8 4}$ & [12] \\
\hline \multirow[t]{5}{*}{ H21S } & D40E & S67T & T80I & $\begin{array}{c}\text { T105I } \\
\text { D113E } \\
\text { L116I }\end{array}$ & 289598452 & [24] \\
\hline & & & T80I & & 289598444 & [24] \\
\hline & D40E & F59I & T80I & $\begin{array}{l}\text { M109T } \\
\text { D113E } \\
\end{array}$ & 289598437 & [24] \\
\hline & $\begin{array}{l}\text { P35S } \\
\text { D40E } \\
\end{array}$ & & $\begin{array}{r}\text { T80I } \\
\text { G87S } \\
\end{array}$ & & 289598430 & [24] \\
\hline & & & T80I & Q113E & 289598423 & [24] \\
\hline $\begin{array}{l}\text { V21S } \\
\text { Y24F }\end{array}$ & & $\begin{array}{l}\text { D64E } \\
\text { I66M } \\
\text { S69A }\end{array}$ & $\begin{array}{c}\text { D77E } \\
\text { T80I }\end{array}$ & V116I & 289598416 & [24] \\
\hline \multirow[t]{4}{*}{ I27V } & & $\begin{array}{c}\text { I55L } \\
\text { Y59I } \\
\text { L66M } \\
\text { G67T }\end{array}$ & T80I & M114T & 289598409 & [24] \\
\hline & F38Y & A67T & $\begin{array}{c}\text { Q79P } \\
\text { T80I } \\
\text { R87S }\end{array}$ & $\begin{array}{l}\text { V105I } \\
\text { I109T } \\
\text { V114T }\end{array}$ & 289598402 & [24] \\
\hline & & $\begin{array}{l}\text { D64E } \\
\text { I66M } \\
\text { N67T } \\
\text { S69A }\end{array}$ & $\begin{array}{c}\text { Q77E } \\
\text { T80I }\end{array}$ & & 289598395 & [24] \\
\hline & $\begin{array}{l}\text { A35S } \\
\text { D40E } \\
\text { Q45P } \\
\end{array}$ & & T80I & $\begin{array}{l}\text { V105I } \\
\text { M109T }\end{array}$ & 289598388 & [24] \\
\hline A21S & $\begin{array}{l}\text { A35S } \\
\text { D40E } \\
\text { H45P }\end{array}$ & N67T & & $\begin{array}{c}\text { T105I } \\
\text { D113E } \\
\text { L116I }\end{array}$ & 289598381 & [24] \\
\hline $\begin{array}{l}\text { T21S } \\
\text { Y24F } \\
\end{array}$ & D40E & T49S & T80I & L116I & 289598374 & [24] \\
\hline \multirow[t]{5}{*}{ M21S } & & $\begin{array}{l}\text { H50P } \\
\text { S67T }\end{array}$ & $\begin{array}{c}\text { Q79P } \\
\text { T80I } \\
\end{array}$ & & 289598367 & [24] \\
\hline & & D64E & $\begin{array}{r}\text { V80I } \\
\text { N87S } \\
\end{array}$ & & 289598360 & [24] \\
\hline & & & $\begin{array}{c}\text { D77E } \\
\text { T80I }\end{array}$ & $\begin{array}{l}\text { D113E } \\
\text { V114T }\end{array}$ & 289598352 & [24] \\
\hline & $\begin{array}{l}\text { F38Y } \\
\text { D40E } \\
\end{array}$ & T49S & $\begin{array}{r}\text { T80I } \\
\text { G87S } \\
\end{array}$ & I114T & 289598345 & [24] \\
\hline & & T49S & T80I & & 289598338 & [24] \\
\hline G21S & & & T80I & & 289598331 & [24] \\
\hline \multirow[t]{5}{*}{ A21S } & & $\begin{array}{c}\text { H57Q } \\
\text { C591 } \\
\text { D64E } \\
\text { N67T } \\
\end{array}$ & T80I & $\begin{array}{l}\text { Q113E } \\
\text { V116I }\end{array}$ & 289598324 & [24] \\
\hline & F38Y & $\begin{array}{l}\text { T49S } \\
\text { I66M } \\
\text { S69A }\end{array}$ & $\begin{array}{r}\text { T80I } \\
\text { E84D }\end{array}$ & L116I & 289598317 & [24] \\
\hline & F38Y & $\begin{array}{l}\text { S50P } \\
\text { D64E } \\
\text { N67T }\end{array}$ & V80I & $\begin{array}{l}\text { L105I } \\
\text { Q113E }\end{array}$ & 289598310 & [24] \\
\hline & & & T80I & & 289598303 & [24] \\
\hline & & H50P & T80I & D113E & 289598296 & [24] \\
\hline A21S & & T49S & T80I & & 289598289 & [24] \\
\hline
\end{tabular}




\begin{tabular}{|c|c|c|c|c|c|c|}
\hline $\begin{array}{l}\text { A21S } \\
\text { Y24F }\end{array}$ & & & T80I & V116I & 289598282 & [24] \\
\hline $\begin{array}{l}\text { S19L } \\
\text { H21S }\end{array}$ & & $\begin{array}{l}\text { D64E } \\
\text { I66M } \\
\text { N67T } \\
\text { S69A } \\
\end{array}$ & T80I & L105I & 289598275 & [24] \\
\hline T21S & $\begin{array}{l}\text { F38Y } \\
\text { P41I }\end{array}$ & & $\begin{array}{l}\text { Q77E } \\
\text { T80I }\end{array}$ & & 289598268 & [24] \\
\hline \multirow[t]{3}{*}{ A21S } & & $\begin{array}{l}\text { A50P } \\
\text { D64E } \\
\text { T66M } \\
\text { N67T } \\
\end{array}$ & $\begin{array}{c}\text { D77E } \\
\text { T80I }\end{array}$ & $\begin{array}{l}\text { D113E } \\
\text { L116I }\end{array}$ & 289598261 & [24] \\
\hline & H45P & D64E & V80I & L116I & 289598254 & [24] \\
\hline & & $\begin{array}{c}\text { F59I } \\
\text { N67T } \\
\end{array}$ & T80I & L116I & 289598247 & [24] \\
\hline \multirow[t]{3}{*}{ T21S } & & S67T & $\begin{array}{l}\text { Q79P } \\
\text { Q80I }\end{array}$ & & 289598240 & [24] \\
\hline & & $\begin{array}{l}\text { D64E } \\
\text { S67T }\end{array}$ & & & 289598233 & {$[24]$} \\
\hline & & & $\begin{array}{l}\text { Q79P } \\
\text { T80I }\end{array}$ & L116I & 289598226 & [24] \\
\hline \multirow[t]{4}{*}{ Y24F } & & & $\begin{array}{c}\text { D77E } \\
\text { T80I }\end{array}$ & V116I & 289598219 & [24] \\
\hline & D40E & $\begin{array}{l}\text { F59L } \\
\text { N67T }\end{array}$ & T80I & $\begin{array}{l}\text { N109T } \\
\text { L116I }\end{array}$ & 289598212 & {$[24]$} \\
\hline & & $\begin{array}{l}\text { D64E } \\
\text { I66M } \\
\text { S69A } \\
\end{array}$ & T80I & & 289598204 & [24] \\
\hline & & & T80I & L116I & 289598196 & [24] \\
\hline A21S & S41A & $\begin{array}{l}\text { L50P } \\
\text { D64E }\end{array}$ & V80I & & 289598189 & [24] \\
\hline \multirow[t]{3}{*}{ V21S } & & & T80I & L116I & 289598182 & [24] \\
\hline & $\begin{array}{l}\text { F38Y } \\
\text { S41A }\end{array}$ & $\begin{array}{l}\text { D64E } \\
\text { I66M } \\
\text { S69A } \\
\end{array}$ & T80I & & 289598175 & [24] \\
\hline & D40E & $\begin{array}{l}\text { T49S } \\
\text { S60L } \\
\text { D64E } \\
\text { L66M } \\
\text { N67T } \\
\text { S69A }\end{array}$ & $\begin{array}{l}\text { Q79P } \\
\text { T80I }\end{array}$ & L116I & 289598168 & [24] \\
\hline H21S & D40E & $\begin{array}{l}\text { D64E } \\
\text { L66M } \\
\text { N67T } \\
\text { S69A }\end{array}$ & T80I & Q113E & 289598161 & [24] \\
\hline \multirow[t]{3}{*}{$\begin{array}{l}\text { N21S } \\
\text { L27V } \\
\end{array}$} & $\begin{array}{l}\text { A35S } \\
\text { F38Y } \\
\end{array}$ & D64E & $\begin{array}{l}\text { V80I } \\
\text { I85V } \\
\end{array}$ & L105I & 289598154 & [24] \\
\hline & F38Y & & T80I & T116I & 289598147 & [24] \\
\hline & F38Y & & $\begin{array}{c}\text { D77E } \\
\text { T80I } \\
\end{array}$ & & 289598140 & [24] \\
\hline \multirow[t]{4}{*}{$\begin{array}{l}\text { Q21S } \\
\text { Y24F }\end{array}$} & T35S & S67T & $\begin{array}{c}\text { Q79P } \\
\text { T80I } \\
\text { T87S }\end{array}$ & $\begin{array}{l}\text { V105I } \\
\text { V114T }\end{array}$ & 289598133 & [24] \\
\hline & & & $\begin{array}{c}\text { Q79P } \\
\text { T80I } \\
\end{array}$ & I114T & 289598126 & [24] \\
\hline & & & T80I & $\begin{array}{c}\text { T105I } \\
\text { D113E } \\
\text { L116I }\end{array}$ & 289598119 & [24] \\
\hline & & D64E & & $\begin{array}{l}\text { T105I } \\
\text { D113E }\end{array}$ & 289598112 & [24] \\
\hline A21S & D40E & $\begin{array}{c}\text { T49S } \\
\text { F59I } \\
\text { N67T } \\
\end{array}$ & $\begin{array}{l}\text { A80I } \\
\text { E83D }\end{array}$ & & 289598104 & [24] \\
\hline
\end{tabular}


chronic and asymptomatic patients and, therefore, may be linked to long term HBV infectivity such as chronic and asymptomatic forms of hepatitis B infection. Therefore, designing a cohort study on acute HBV infected patients in relation to $\mathrm{HBc}$ gene mutations and following the outcomes of infections could help find the mutations responsible for induction of long term hepatitis B infection. Previous studies reported that the HBV genotype can affect the outcome of infection. Interestingly, the D genotype is the unique prevalent HBV genotype in Iran [13]; hence, the genotype does not appear to affect the outcome of disease in Iran, rather, and it would appear that the mutations reported here can be considered more important for disease progression. In addition, based on the high prevalence of OBI in Iranian blood donors [33], evaluation of these mutations in the long term forms of hepatitis B infection would be of immense value.

\section{Acknowledgement:}

This project was supported by a grant from the Rafsanjan University of Medical Sciences and the authors declare no conflict of interest regarding the review article.

\section{Declaration of Interest:}

The authors of this manuscript have no invested interests in products described or used in this article. The authors have no conflicts of interest.

\section{References:}

1. Assar S, Arababadi MK, Ahmadabadi BN, Salehi M, Kennedy D. Occult Hepatitis B virus (HBV) infection: a global challenge for medicine. Clin Lab 2012;58(11-12):1225-30.

2. Babaei M, Pourfarzi F, Yazdanbod A, et al. Gastric cancer in Ardabil, Iran--a review and update on cancer registry data. Asian Pac J Cancer Prev 2010;11(3):595-9.

3. Arababadi MK, Pourfathollah AA, Jafarzadeh A, Hassanshahi G, Rezvani ME. Association of exon 9 but not intron 8 VDR polymorphisms with occult HBV infection in south-eastern Iranian patients. J Gastroenterol Hepatol 2009 Jan;25(1):90-3.

4. Yuen MF, Lee CK, Wong DK, et al. Prevalence of occult hepatitis B infection in a highly endemic area for chronic hepatitis B: a study of a large blood donor population. Gut 2011 Oct;59(10): 1389-93.

5. Yoshikawa A, Gotanda Y, Minegishi K, et al. Lengths of hepatitis B viremia and antigenemia in blood donors: preliminary evidence of occult (hepatitis B surface antigen-negative) infection in the acute stage. Transfusion 2007 Jul;47(7):1162-71.

6. Plymoth A, Viviani S, Hainaut P. Control of hepatocellular carcinoma through hepatitis B vaccination in areas of high endemicity: perspectives for global liver cancer prevention. Cancer Lett 2009 Dec 1;286(1):15-21.
7. Arababadi MK, Hassanshahi G, Pourfathollah AA, Zarandi ER, Kennedy D. Post-Transfusion Occult Hepatitis B (OBI): A Global Challenge for blood recipients and health authorities. Hepat Mon 2011; Article in press.

8. Arababadi MK, Pourfathollah AA, Jafarzadeh AA, Hassanshahi G. Serum levels of Interleukin (IL)-10 and IL-17A in occult HBV infected South-East Iranian patients. Hepat Mon 2010;10(1):31-5.

9. Arababadi MK, Ahmadabadi BN, Kennedy D. Current information on the immunological status of occult hepatitis B infection. Transfusion 2012; Article in press.

10. Frelin L, Wahlstrom T, Tucker AE, et al. A mechanism to explain the selection of the hepatitis e antigen-negative mutant during chronic hepatitis B virus infection. J Virol 2009 Feb;83(3):137992.

11. Assar S, Arababadi MK, Mohit M, et al. T helper and B cell escape mutations within the $\mathrm{HBc}$ gene in patients with asymptomatic HBV infection: a study from the South-Eastern region of Iran. Clin Lab 2012;58(1-2):53-60.

12. Mohebbi SR, Amini-Bavil-Olyaee S, Zali N, et al. Characterization of hepatitis B virus genome variability in Iranian patients with chronic infection, a nationwide study. J Med Virol 2012 Mar;84(3):414-23.

13. Eftekhari Y, Arababadi MK, Hakimi H, Zarandi ER. Common HBV genotype in Southeastern Iranian patients. Arch Iran Med 2010;13(2):147-9.

14. Arababadi MK, Pourfathollah AA, Jafarzadeh A, Hassanshahi G, Mohit M, Hajghani M, et al. Peripheral blood CD8+ T cells CCR5 expression and its $\Delta 32$ mutation in Iranian patients with occult hepatitis B infection. LabMedicine 2010;41:226-30.

15. Livingston BD, Crimi C, Fikes J, Chesnut RW, Sidney J, Sette A Immunization with the HBV core 18-27 epitope elicits CTL responses in humans expressing different HLA-A2 supertype molecules. Hum Immunol 1999 Nov;60(11):1013-7.

16. Sendi H, Mehrab-Mohseni M, Shahraz S, et al. CTL escape mutations of core protein are more frequent in strains of $\mathrm{HBeAg}$ negative patients with low levels of HBV DNA. J Clin Virol 2009 Nov;46(3):259-64.

17. Medzhitov R, Shevach EM, Trinchieri G, et al. Highlights of 10 years of immunology in Nature Reviews Immunology. Nat Rev Immunol 2011 Oct;11(10):693-702.

18. Deenick EK, Ma CS. The regulation and role of T follicular helper cells in immunity. Immunology 2011 Dec;134(4):361-7.

19. Milich DR. Influence of T-helper cell subsets and crossregulation in hepatitis B virus infection. J Viral Hepat 1997;4 Supp1 2:48-59.

20. Breitfeld D, Ohl L, Kremmer E, et al. Follicular B helper T cells express CXC chemokine receptor 5, localize to B cell follicles, and support immunoglobulin production. J Exp Med 2000 Dec 4, 192(11):1545-52.

21. King C, Sprent J. Emerging cellular networks for regulation of T follicular helper cells. Trends Immunol 2012 Feb;33(2):59-65.

22. Jung MC, Pape GR. Immunology of hepatitis B infection. Lancet Infect Dis 2002 Jan;2(1):43-50.

23. Chen W, Shi M, Shi F, et al. HBcAg-pulsed dendritic cell vaccine induces Th1 polarization and production of hepatitis B virusspecific cytotoxic T lymphocytes. Hepatol Res 2009 Apr;39(4): 355-65. 
24. Garmiri P, Rezvan H, Abolghasemi H, Allain JP. Full genome characterization of hepatitis B virus strains from blood donors in Iran. J Med Virol 2011 Jun;83(6):948-52.

25. Barboza L, Salmen S, Peterson DL, et al. Altered T cell costimulation during chronic hepatitis B infection. Cell Immunol 2009; 257(1-2):61-8.

26. Arababadi MK, Pourfathollah AA, Jafarzadeh A, et al. Non-association of IL-12 +1188 and IFN-gamma +874 polymorphisms with cytokines serum level in occult HBV infected patients. Saudi J Gastroenterol 2011 Jan-Feb;17(1):30-5.

27. Huang LM, Lu CY, Chen DS. Hepatitis B virus infection, its sequelae, and prevention by vaccination. Curr Opin Immunol 2011 Apr;23(2):237-43.

28. Chisari FV, Isogawa M, Wieland SF. Pathogenesis of hepatitis B virus infection. Pathol Biol (Paris) 2010 Aug;58(4):258-66.

29. Kamili S. Infectivity and vaccination efficacy studies in animal models of HBV S and pol gene mutants. Antivir Ther 2010;15(3 Pt B):477-85.

30. Ponde RA, Cardoso DD, Ferro MO. The underlying mechanisms for the 'anti-HBc alone' serological profile. Arch Virol $2010 \mathrm{Feb}$; 155(2):149-58.

31. Moradi A, Zhand S, Ghaemi A, Javid N, Tabarraei A. Mutations in the $\mathrm{S}$ gene region of hepatitis B virus genotype D in Golestan Province-Iran. Virus Genes 2012 Jun;44(3):382-7.
32. Svicher V, Cento V, Salpini R, et al. Role of hepatitis B virus genetic barrier in drug-resistance and immune-escape development. Dig Liver Dis 2011 Dec;43(12):975-83.

33. Arababadi MK, Pourfathollah AA, Jafarzadeh A, Hassanshahi G. Decreased expression of CCR5 on the NK cells in occult HBV infected patients. LabMedicine 2010;41(12):735-8.

34. Amini-Bavil-Olyaee S, Sarrami-Forooshani R, Adeli A, et al. Complete genomic sequence and phylogenetic relatedness of hepatitis B virus isolates from Iran. Journal of Medical Virology $2005 \mathrm{Jul} ; 76(3): 318-26$.

35. Amini-Bavil-Olyaee S, Sarrami-Forooshani R, Mahboudi F, et al. Genotype characterization and phylogenetic analysis of hepatitis B virus isolates from Iranian patients. J Med Virol 2005 Feb; 75(2):227-34.

36. Mohebbi SR, Amini-Bavil-Olyaee S, Zali N, et al. Molecular epidemiology of hepatitis B virus in Iran. Clin Microbiol Infect 2008 Sep;14(9):858-66.

\section{Correspondence:}

Dr. Tayebeh Mirzaei

Dept of Nursing

School of Nursing and Midwifery

Rafsanjan University of Medical Sciences

Rafsanjan, Iran

Tel.: $\quad+983915234003-5$

Fax: $\quad+983915225209$

Email: t.mirzaei@rums.ac.ir 